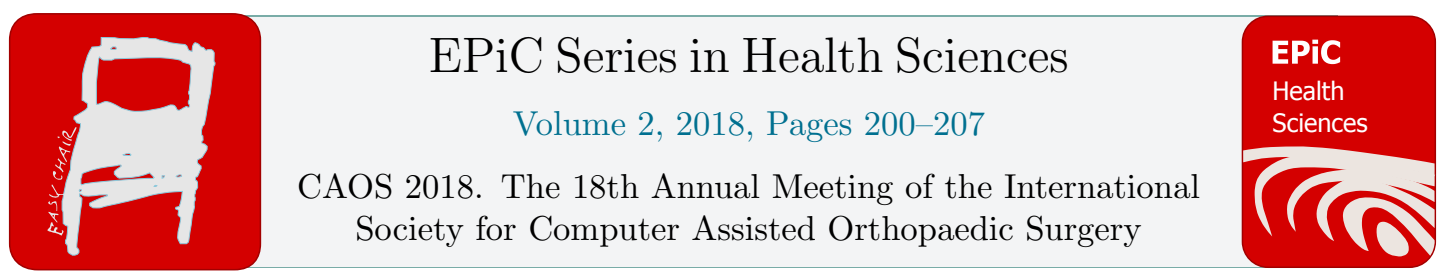

\title{
MEASUREMENT OF INTRAOPERATIVE RANGE OF MOTION OF TOTAL HIP ARTHROPLASTY USING AN INERTIAL MEASUREMENT UNIT BASED SMART TRIAL SYSTEM: AN IN VITRO VALIDATION EXPERIMENT
}

\author{
Hao Tang ${ }^{1}$, MD, MRCS, Yixin Zhou ${ }^{1 *}$, MD, Baojun $\mathrm{Mai}^{2}$, Binjie Zhu², \\ Ping Chen ${ }^{2}$, Yujia Fu ${ }^{2}$ \\ $1 *$ Department of Orthopaedic Surgery, Beijing Jishuitan Hospital, Fourth Clinical College of Peking \\ University, Beijing, China; orthoyixin@yahoo.com; \\ ${ }^{2}$ Beijing Yiemed Medical Technology Co. Ltd, Beijing, China;
}

\begin{abstract}
Intraoperative detection of hip Range of Motion ( ROM ) is the basis for prediction of postoperative functional limits allowed for patients' daily living. Although computer navigation system for Total Hip Arthroplasty ( THA ) has improved the accuracy of intraoperative ROM evaluation, it has not been able to gain popularity because of its complex and time-consuming procedures. We preliminarily developed a Inertial Measurement Unit based Hip Smart trial system ( IMUHS ) that is easy and simple to use. An in vitro validation experiment was conducted using radiopaque sawbones with imaging measurement method as the reference standard. The validity of measuring ROM in the three axes was as follows: $4.36 \pm 4.93^{\circ}$ for flexion and extension, $1.75 \pm 5.21^{\circ}$ for adduction and abduction, and $6.42 \pm 4.32^{\circ}$ for internal and external rotation( $\left.p=0.059 \sim 0.550\right)$. The ICC values of measuring ROM in all three axes were 1.00. As measuring ROM is the basis for evaluating direction of impingement, subluxation and dislocation, the IMUHS is a promising development direction of THA computer assisted surgery.
\end{abstract}


Measurement of Intraoperative Range of Motion of Total Hip Arthroplasty Using an ... H. Tang et al.

\section{Introduction}

Total Hip Arthroplasty is on rapid increase worldwide. Dislocation is a major postoperative complication leading to failure of the procedure. Measuring Range of Motion ( ROM ) is the basis for intraoperative evaluating direction of impingement, subluxation and dislocation.

Traditionally, intraoperative measurement of ROM was done by surgeon's subjective assessment. Optics based navigation systems improved the accuracy of ROM detection, but was expensive, time-consuming, and relied on complex hardware system, which made it unable to be widely used in every patient. It was reported that currently navigation was used in only $1 \%-3 \%$ of procedures.(Paprosky \& Muir, 2016; Xu et al., 2014)

Inertial Measurement Unit is a collection of sensors that could be used to measure the posture of bodies.(Fasel, Sporri, Schutz, Lorenzetti, \& Aminian, 2017) In order to accurately measure the intraoperative ROM, which is the basis for computer detection of impingement, subluxation and dislocation, we preliminarily developed a Inertial Measurement Unit based Hip Smart trial system ( IMUHS ) that was aimed to be easy and simple to use, and not dependent upon complex optic systems.

An in vitro experiment was done to examine the validity and reliability of measuring ROM using the IMUHS system prototype.

\section{Materials and Methods}

\subsection{Inertial Measurement Unit based Hip Smart trial system (IMUHS )}

The Inertial Measurement Unit based Hip Smart trial system (IMUHS) proposed in this paper was composed of measurement hardware in a femoral head trial and application software in a terminal computer. The head trial and computer were connected via a USB cable. ( Fig. 1 ) 
In the head trial, a 3-axis accelerometer, 3-axis gyroscope and 3-axis magnetometer were applied to building an IMU system, Kalman filtering and 9-axis fusion algorithm were employed to computing the rotation angle on a microprogrammed control unit (MCU).

With the real time measuring data, we can read and record the ROM on the smart trial software. The ROM was defined as rotation angles into the local femoral coordinate system from the neutral position (Miki H, Kyo T, Sugano N, 2012). The first rotation (flexion or extension) is moved around the z-axis of femur, and the second (abduction or adduction) and third (external or internal rotation) rotations are respectively around the $\mathrm{y}$-axis and $\mathrm{x}$-axis.

\subsection{Sawbone range of motion measurement and CT Scanning}

To simulate the total hip arthroplasty, we employed a radiopaque sawbone pelvis and femur, implanted a $60 \mathrm{~mm}$ trial cup into the right acetabulum after reaming, and a trial femoral stem into to the femoral canal after broaching using surgical instrument ( Depuy, Warsaw, IN). The IMUHS $36 \mathrm{~mm}$ diameter trial head was then assembled onto the femoral trial.

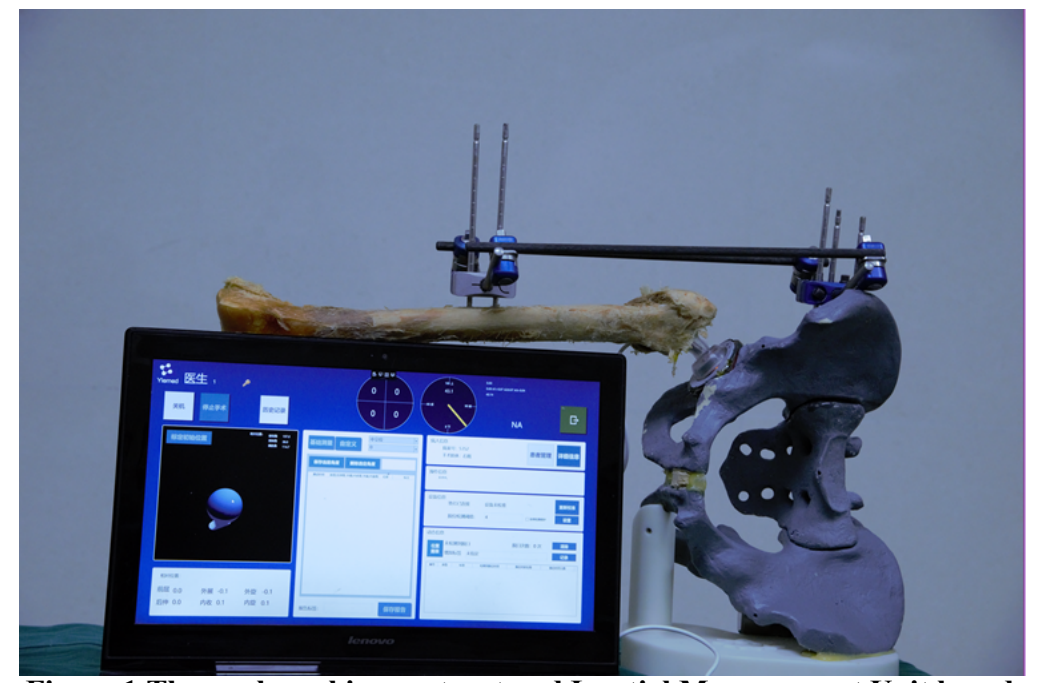

Figure 1 The sawbone hip construct and Inertial Measurement Unit based Hip Smart trial system ( IMUHS ) in neutral position 
Measurement of Intraoperative Range of Motion of Total Hip Arthroplasty Using an ... H. Tang et al.

The pelvis was fixed onto a scaffold, which was designed and printed using additive manufacture technique, and ensured that the anterior pelvic plane was perpendicular to the ground, and the construct was then fixed on to a horizontal experiment table surface.

The sawbone hip was put into a neutral position, and fixed with an external fixator system ( Stryker Orthopedics, Mahwah, NJ ). The neutral position was calibrated and captured by the IMUHS system, and then scanned by a Computed Tomography ( CT ) system ( SOMATOM Emotion, Siemens, Munich, Germany ). Scanning from $2.0 \mathrm{~cm}$ above the superior rim of pelvis to $2.0 \mathrm{~cm}$ distal to the distal femoral articular surface, axial sections of $1.5 \mathrm{~mm}$ thickness were obtained according to the standard protocol in our hospital $120 \mathrm{kV}, 250 \mathrm{~mA}$ and $145 \mathrm{mAs}$, field of view dimension $41.0 \mathrm{~cm}$, rotation time 0.5 seconds, matrix size $512 \times 512$ ). The sawbone hip was then put into 15 different positions and the process above was repeated to obtain the CT data in Digital Imaging and Communications in Medicine format ( DICOM ) and IMUHS measurement data for each position. The IMUHS measurement was done for three times for each rotated position to examine the reliability.

\subsection{Image Based Range of Motion Measurement}

The ROM data was measured for each different position as compared to the original neutral position from the DICOM data using mimics 10.0 software (Materialise, Leuven, Belgium). The registration function was employed to first register the pelvis of the to-be-measured position onto the neutral position, and the ROM was then measured by using the reposition function to rotate the femur in neutral position to superimpose the femur in the to-be-measured position. Each position was measured by 3 different authors, and the average values of flexion/extension, abduction/adduction, and internal /external rotation measured by mimics were used as the reference standard for IMHUS results. 


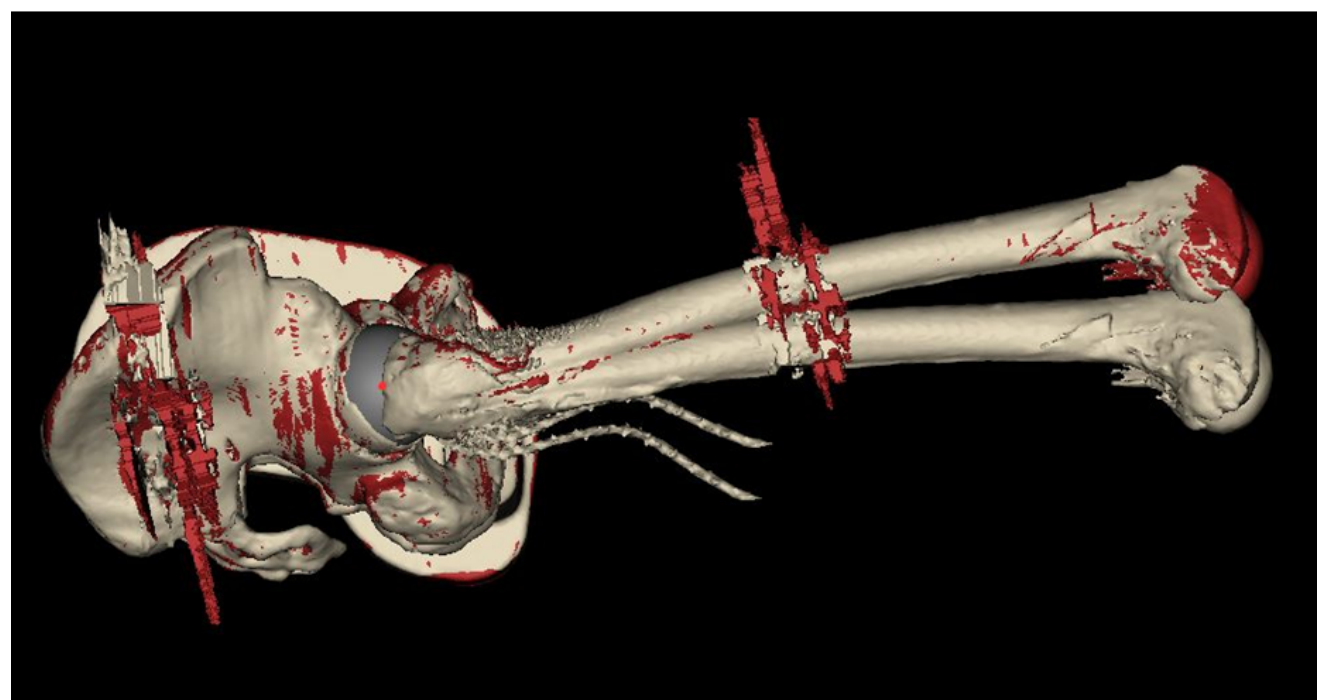

Figure 2 The measurement of Range of Motion in the mimics 10.0 software using the Computed tomography data. Red bone is the position where femur rotated a certain degree, and white bone is the position of femur at the neutral position (below) and the position rotated to superimpose the red femur. The pelvises ( white and red ) were registered to overlap each other.

With the image based ROM measurement as the reference standard, we calculated the validity, reliability of measuring ROM by the IMUHS system with matched t-test and Interclass Correlation Coefficient ( ICC ). All statistical analysis was done using SPSS software ( version 15.0; IBM, Armonk, NY, USA ), and the level of significance was set at $\mathrm{p}<0.05$.

\section{Results}

The validity of measuring ROM in the three axes was as follows: $4.36 \pm 4.93^{\circ}$ for flexion and extension, $1.75 \pm 5.21^{\circ}$ for adduction and abduction, and $6.42 \pm$ $4.32^{\circ}$ for internal and external rotation. (Table 1.)

The ICC values of measuring ROM in the three axes was 1.00 for all rotation axes. 
Measurement of Intraoperative Range of Motion of Total Hip Arthroplasty Using an ... H. Tang et al.

\begin{tabular}{lccc}
\hline \multicolumn{1}{c}{ Dimension } & \multicolumn{3}{c}{ Difference } \\
\cline { 2 - 4 } & Mean & SD & p value \\
\hline Flexion/Extension & 4.36 & 4.93 & 0.168 \\
Adduction/Abduction & 1.75 & 5.21 & 0.550 \\
Internal/External rotation & 6.42 & 4.32 & 0.059 \\
\hline
\end{tabular}

Table 1. Validity results. $\mathrm{SD}=$ Standard Deviation

\section{Discussion}

The Inertial Measurement Unit based Hip Smart trial system ( IMUHS ) was the foundation for developing further intraoperative smart trial system including the functions of detecting direction of impingement, subluxation and dislocation.

Most accuracy studies focused on the accuracy of component positioning during surgeries. Very few literature reported the accuracy of optical navigation system in measuring ROM. Kendoff et al. Reported that a rotational deviation of 3 degrees was obtained with a noninvasive optic tracker system.(Kendoff et al., 2007) In comparison with traditional optics based navigation systems, although the validity and reliability as we measured were not that high, this system features other advantages: it's less invasive as no extra trackers on bones were needed; it's more convenient and easy to use; and it does not require complex and expensive navigation hardware system. (Inaba, Kobayashi, Ike, Kubota, \& Saito, 2016; Jassim, Benjamin-Laing, Douglas, \& Haddad, 2014; Xu et al., 2014)

IMU based tools has been introduced in to the arthroplasty field recently. Several accelerator based systems has been developed for total knee arthroplasty and achieved good results.(Gharaibeh, Solayar, Harris, Chen, \& MacDessi, 2017) However, very few IMU based navigation system for THA has been developed. Silvio reported that they developed an IMU based navigation system for 
periacetabular osteotomy and obtained a mean difference of within 4 degrees. To our knowledge, there has been no reports about a intro-articular IMU based smart sensor been developed to measure the ROM.

According to Our data, the accuracy in the flexion/extension and adduction/abduction planes were relatively lower compared with that in the internal/external rotation plane, with the image based measurement results as the reference standard. This might be because the local disturbance of magnetic field caused by the CT machine affects the internal/external rotation results more than the other two dimensions.

We understand that this research has several limitations. First, the in vitro study could not fully simulate the intraoperative situation, as there were no soft tissue nor body fluid surrounding the sawbone, and the local environment of operation theaters may defer from the laboratory. Second, THA navigation system was not used in the study as no THA navigation system was available to us yet. Further study including a navigation system as reference is needed. Third, the IMU sensors' accuracy was susceptible to environmental electromagnetism disturbances, therefore strong magnetic field alterations should be avoided during its application.

The IMUHS is a promising sensor system of THA computer assisted surgery.

\section{Reference}

Fasel, B., Sporri, J., Schutz, P., Lorenzetti, S., \& Aminian, K. (2017). An Inertial Sensor-Based Method for Estimating the Athlete's Relative Joint Center Positions and Center of Mass Kinematics in Alpine Ski Racing. Front Physiol, 8, 850. doi: 10.3389/fphys.2017.00850

Gharaibeh, M. A., Solayar, G. N., Harris, I. A., Chen, D. B., \& MacDessi, S. J. (2017). Accelerometer-Based, Portable Navigation (KneeAlign) vs Conventional Instrumentation for Total Knee Arthroplasty: A Prospective Randomized Comparative Trial. [Comparative Study

Randomized Controlled Trial]. J Arthroplasty, 32(3), 777-782. doi: 10.1016/j.arth.2016.08.025

Inaba, Y., Kobayashi, N., Ike, H., Kubota, S., \& Saito, T. (2016). The current status and future prospects of computer-assisted hip surgery. [Lectures]. J Orthop Sci, 21(2), 107-115. doi: 10.1016/j.jos.2015.10.023 
Measurement of Intraoperative Range of Motion of Total Hip Arthroplasty Using an ... H. Tang et al.

Jassim, S. S., Benjamin-Laing, H., Douglas, S. L., \& Haddad, F. S. (2014). Robotic and navigation systems in orthopaedic surgery: how much do our patients understand? Clin Orthop Surg, 6(4), 462-467. doi: 10.4055/cios.2014.6.4.462

Kendoff, D., Bogojevic, A., Citak, M., Maier, C., Maier, G., Krettek, C., \& Hufner, T. (2007). Experimental validation of noninvasive referencing in navigated procedures on long bones. [Validation Studies]. J Orthop Res, 25(2), 201-207. doi: 10.1002/jor.20318

Paprosky, W. G., \& Muir, J. M. (2016). Intellijoint HIP((R)): a 3D mini-optical navigation tool for improving intraoperative accuracy during total hip arthroplasty. Med Devices (Auckl), 9, 401-408. doi: 10.2147/MDER.S119161

Xu, K., Li, Y. M., Zhang, H. F., Wang, C. G., Xu, Y. Q., \& Li, Z. J. (2014). Computer navigation in total hip arthroplasty: a meta-analysis of randomized controlled trials. [Meta-Analysis

Research Support, Non-U.S. Gov't]. Int J Surg, 12(5), 528-533. doi: 10.1016/j.ijsu.2014.02.014

Miki H, Kyo T, Sugano N. Anatomical hip range of motion after implantation during total hip arthroplasty with a large change in pelvic inclination[J]. Journal of Arthroplasty, 2012, 27(9):16411650.

\section{DISCLOSURES}

Dr. Yixin Zhou and Dr. Hao Tang collaborated with the Beijing Yiemed Medical Technology Co. Ltd to develop this system and conducted the validation experiment.

-- end of paper template -- 